\title{
Tight Bounds for Visibility Matching of $f$-Equal Width Objects
}

\author{
David Rappaport \\ School of Computing, Queen's University \\ Kingston, Ontario, K7L 3N6, CANADA
}

\begin{abstract}
Let $s$ denote a compact convex object in $\mathbb{R}^{2}$. The $f$-width of $s$ is the perpendicular distance between two distinct parallel lines of support of $s$ with direction $f$. A set of disjoint convex compact objects in $\mathbb{R}^{2}$ is of equal $f$-width if there exists a direction $f$ such that every pair of objects have equal $f$-width. A visibility matching, for a set of equal $f$-width objects is a matching using non-crossing lines of site in the visibility graph of the set. In this note we establish tight bounds on the size of a maximal visibility matching for a set of $f$-equal width objects by showing that $\left\lfloor\frac{2 n}{3}\right\rfloor \leq h(n) \leq \frac{2 n}{3}$.
\end{abstract}

\section{Introduction}

Let $S$ denote a set of non-intersecting compact convex objects in $\mathbb{R}^{2}$. We say that two objects $a$ and $b$ from the set $S$ see each other if there exists a straight line segment $l$ with one point in $a$ and one point in $b$ such that $l$ lies in the complement of $S-\{a, b\}$. We call such a line segment a line of sight. The visibility graph of $S$, denoted by $\operatorname{Vis}(S)$, associates a vertex to each object of $S$, and an edge between two vertices if and only if the associated objects see each other.

The combinatorial structure of the visibility graph for sets in $\mathbb{R}^{2}$ has been studied extensively. Some results on the combinatorial structure of the visibility graph of line segments can be found in $[1,2,4,6,8]$.

In [4] the notion of set of $f$-equal width objects is introduced. Let $s$ denote a compact object in $\mathbb{R}^{2}$. The $f$-width of $s$ is the perpendicular distance between two distinct parallel lines of support of $s$ with direction $f$. A set of compact objects in $\mathbb{R}^{2}$ is of $f$-equal width if there exists a direction $f$ such that every pair of objects have $f$-equal width. A visibility matching, for a set of $f$-equal width objects is a matching using non-crossing lines of sight in the visibility graph of the set. Hosono [3] has shown that size of a maximal visibility matching of $2 n$ $f$-equal width objects $h(n)$ satisfies the inequalities $\left\lfloor\frac{2 n}{3}\right\rfloor \leq h(n) \leq\left\lfloor\frac{4 n}{5}\right\rfloor$. In this note we establish tight bounds on the size of a maximal visibility matching for a set of $f$-equal width objects by showing that $\left\lfloor\frac{2 n}{3}\right\rfloor \leq h(n) \leq \frac{2 n}{3}$.

Some related results regarding the visibility graph of disjoint convex objects appear in a paper by Hosono, Meijer, and Rappaport [5] where it is shown that a set of translates of disjoint convex bodies admit a Hamilton path. In [7] it shown that the visibility graph of a set of disjoint congruent discs is Hamiltonian. 


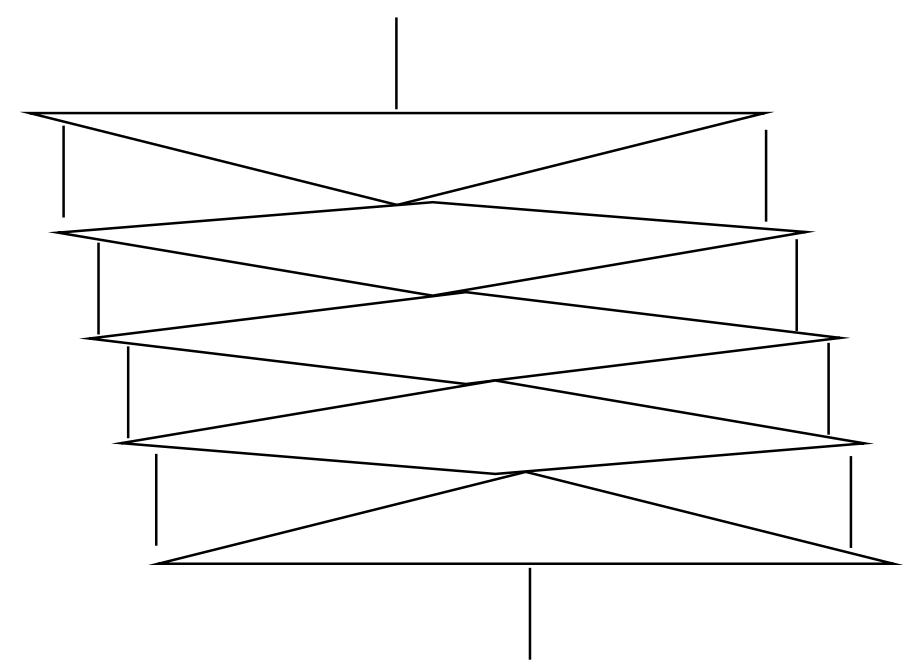

Fig. 1. This example leads to a maximal matching of at most $\frac{2 n}{3}$.

\section{Lower Bound}

Throughout we assume that $S$ is a set of $f$-equal width objects and $f$ is horizontal.

Let $\Gamma$ denote an arbitrary compact subset of $\mathbb{R}^{2}$. We say that a line $l$ supports $\Gamma$ if $l \cap \Gamma \neq \emptyset$, and a closed halfplane bounded by $l$ contains $\Gamma$. If a point $\gamma \in \Gamma$ is contained in $l$ then we say that $\gamma$ is extreme.

For an element $s \in S$, let $\operatorname{NoRTH}(s)$ be used to denote a horizontal line of support passing through an extreme point in $s$ with maximum $y$ coordinate, and let $\operatorname{South}(s)$ be used to denote a horizontal line of support passing through an extreme point in $s$ with minimum $y$ coordinate. Let $s, t \in S$, then $s<t$ if $\operatorname{North}(s)$ is above $\operatorname{NorTh}(t)$, or when $s$ and $t$ are supported by the same horizontal line, then $s$ is to the left of $t$. We use $<_{\min }(S)$ to denote the least element of $S$ using the < ordering.

Consider a set $S$ of $f$-equal width objects. We can partition the set $S$ based on the sweep of a horizontal line . Let $S_{0}$ denote the subset of $S$ intersected $\operatorname{SoUTH}\left(<_{\min }(S)\right)$. Let $\Sigma_{i}=S-S_{0} \cup \ldots \cup S_{i-1}$. We give a recursive definition for $S_{i}$ the subset of $S$ intersected by $\operatorname{SoutH}\left(<_{\min }\left(\Sigma_{i}\right)\right)$.

Observe that if the cardinality of the smallest $S_{i}$ is 2 or more then we obtain our lower bound by simply matching objects within the same subset of our partition. It remains to settle the issue when there are subsets of cardinality 1 .

Let $S_{j-1}, j \leq n-1$ be a subset of cardinality 1, whose only element is called $s$. If $S_{j}$ is of cardinality 1 then there is a line of sight between the element in $S_{j}$ and $s$ and they can be matched. Furthermore, if the cardinality of $S_{j}$ is 2 or 4 or more then the matched objects in $S_{j}$ overcome the unmatched $s$ and the bound 
is achieved. Thus consider the case where $S_{j}$ is of cardinality 3 , and let a left to right labelling of the objects of $S_{j}$ be $a, b, c$. If $s$ sees $a$ or $c$ we match all of the objects in the two sets. For example $s$ sees $a$ and since there is a horizontal line intersecting $a, b, c$ and no other element of $\Sigma_{j}$ then $b$ must see $c$. Suppose on the other hand that $s$ does not see either $a$ or $c$. There is a horizontal line that intersects $s$ and no other elements of $\Sigma_{j-1}$ so $s$ must see at least one object in $S_{j}$ namely $b$. However, this implies that $b$ is higher than both $a$ or $c$ (otherwise $s$ could see $a$ or $c$ ) so there is a horizontal line of sight between $a$ and $c$. Therefore we match all of the objects in the union of $S_{j-1}$ and $S_{j}$. Finally we have the case where $S_{n-1}$ is of cardinality 1 . In this event the best lower bound we can guarantee is $2 n / 3-1$.

\section{Upper Bound}

We give a construction that achieves the stated upper bound. Consider $3 N f$ equal width objects, as shown in Figure 1. The objects are a collection of $2 \mathrm{~N}$ vertical unit length line segments called bars and $N$ gems. Each gem is built upon a skeleton made up of a unit length vertical line segment and a horizontal line segment. See Figure 2.

Let $n=N-1$ We can number the gems $g_{0}, \ldots, g_{n}$ from top to bottom. The horizontal segment of $g_{i}$ is located a distance of $i / n$ from the top of the vertical segment. Let $c$ be a positive constant. Each horizontal segment of the gems skeleton is of length $2 \mathrm{cn}$ and is bisected by the vertical. The gems are placed one below the other, such that $g_{i}$ is slightly to the right of $g_{i-1}$, and pushed up so that the gems are almost touching. That is there exists small positive values $\epsilon$ and $\epsilon_{0}, \epsilon_{1}, \ldots, \epsilon_{n}$ so that the top of $g_{i}$ is positioned at coordinate $\left(i \epsilon,-i+\epsilon_{i}\right)$. We put a bar above the top gem and a bar below the bottom gem, and respectively call them top and bot. We can now fit the remaining $2 n$ bars between the gems on the left and on the right. We refer to these bars as $l_{1} \ldots l_{n}$ and $r_{1} \ldots r_{n}$. There exists a positive value $\delta$ such that $l_{i}$ can be placed directly above the left end of $g_{i}$ so that the vertical gap between the segment and the boundaries of $g_{i-1}$ and $g_{i}$ is at least $\delta$. On the right we place $r_{i}$ directly below the right extreme of $g_{i-1}$ again maintaining a gap of at least $\delta$ between the bar and its neighbouring gems. We prove that this construction is possible without overlapping any of the objects.

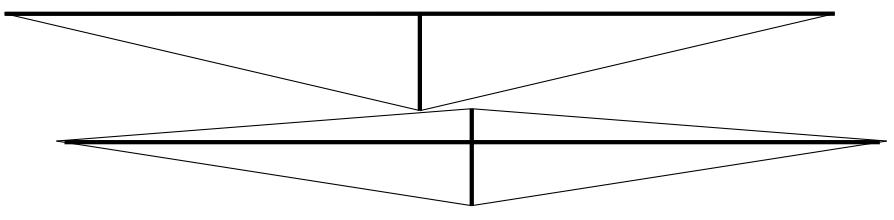

Fig. 2. The anatomy of a gem with its skeleton in bold lines. 
Lemma 1. The vertical distance between the left extreme of $g_{i}$ and the boundary of $g_{i-1}$ is at least $1+2 \delta$

Proof: Consider the distance between the two consecutive horizontal segments, say between $g_{i-1}$ and $g_{i}$. This distance is at least $1+1 / n-\epsilon_{i}$. Using similar triangles observe that the vertical distance of the boundary of $g_{i-1}$ from the horizontal line segment is at most

$$
\frac{\epsilon\left(1-\frac{i}{n}\right)}{c n} \leq \frac{\epsilon}{c n}
$$

Also observe that

$$
\epsilon_{i}<\frac{\epsilon}{c n}, i=0, \ldots n \text {. }
$$

Therefore the vertical distance between the boundary of $g_{i-1}$ and the left extreme of $g_{i}$ is at least $1+\frac{1}{n}-\frac{2 \epsilon}{c n}$. If we set $c$ to $4 \epsilon$ we have the desired result, then $\delta$ becomes $1 / 4 n$.

We claim that the visibility graph of such a set of objects is isomorphic to a graph $G$ with vertex set $\left\{g_{0}, g_{1}, \ldots, g_{n}, l_{1}, l_{2}, \ldots, l_{n}, r_{1}, r_{2}, \ldots, r_{n}\right.$, top, bot, $\}$, and edges $\left\{\left(l_{i}, g_{i}\right),\left(l_{i}, g_{i-1}\right),\left(r_{i}, g_{i}\right),\left(r_{i}, g_{i-1}\right),\left(g_{i}, g_{i-1}\right)\right.$, (top, $\left.g_{0}\right)$, (bot, $\left.\left.g_{n}\right)\right\}$, for $i=1 \ldots n$. See Figure 3 .

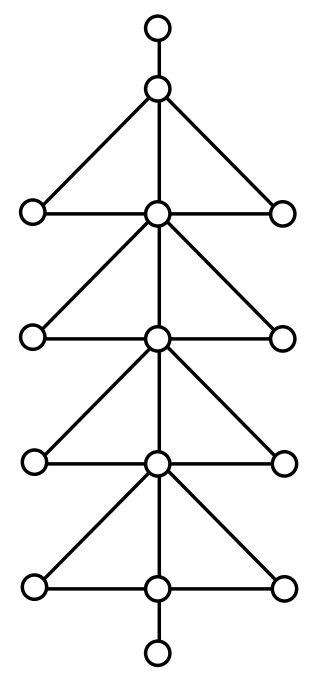

Fig. 3. The visibility graph for the objects.

Theorem 1. $\operatorname{Vis}(S)$ is isomorphic to $G$.

Proof: The inclusion of the prescribed edges is not in question and can easily be verified. We show that no other edges exist in the visibility graph. It is 
clear that top only sees $g_{0}$ and bot only sees $g_{n}$. Observe that the right and left extremes of the gems are on a straight line (they could also be put on the arc of a parabola, to avoid using degeneracies in our argument) it is clear the $g_{i}$ sees $g_{i-1}$ and $g_{i+1}$ and no other gems. Similarly the bars $r_{i}$ see no other $r_{j}$ and no $l_{j}$, for $i \neq j$. Furthermore we claim that $l_{i}$ cannot see $r_{i}$. The gap between $g_{i}$ and $g_{i+1}$ can be made as small as one wishes. Thus the only lines of sight that leak through the space between $g_{i}$ and $g_{i+1}$ are arbitrarily close to the boundaries of the respective gems. To be specific, the distance between gems effects the angle between the sight leak and the boundary of $g_{i}$. Thus we can make this angle as small as we like. On the other hand we also have control over the size of $\delta$ which is guaranteed to be some positive value. See Figure 4.

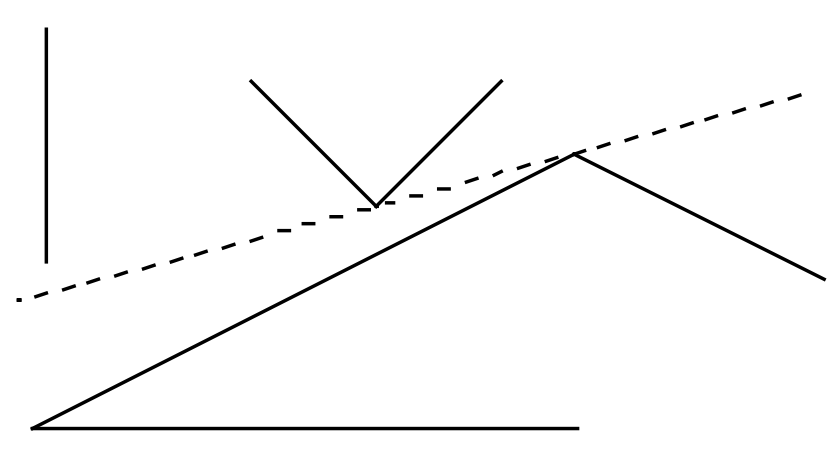

Fig. 4. Detail of a pair of gems and the amount of sight leak between them.

Since the bars are of distance at least $\delta$ from the gems there is no line of sight between any $r_{i}$ and $l_{i}$. Thus we have shown that $\operatorname{Vis}(S)$ is isomorphic to $G$.

It is now a routine matter to verify that a maximal matching in the derived visibility graph saturates $2 N$ of the $3 N$ objects.

\section{References}

1. P. Bose, G. Toussaint: Growing a tree from its branches, Journal of Algorithms, 19 (1995)86-103.

2. M. Hoffmann and C. S. T'oth, Segment endpoint visibility graphs are Hamiltonian, In Proc. 13th Canadian Conference on Computational Geometry (CCCG '01), Waterloo(ON), Canada, (2001)109-112, To appear in Computational Geometry: Theory and Applications, 2003.

3. K. Hosono On an estimate of the size of the maximum matching for a disjoint family of compact convex sets in the plane, Discrete Appl. Math. 113 (2001) 291-298.

4. K. Hosono and K. Matsuda, On the perfect matching of disjoint convex sets by line segments, Discrete Appl. Math. 118 (2002) 223-238. 
5. K. Hosono, H. Meijer, D. Rappaport, On the visibility graph of convex translates, Discrete Appl. Math. 113 (2001) 195-210.

6. A. Mirzaian, Hamiltonian triangulations and circumscribing polygons of disjoint line segments, Comput. Geom. Theory Appl., 2, 1 (1992) 15-30.

7. D. Rappaport The visibility graph of congruent discs is Hamiltonian, to appear in Computational Geometry: Theory and Applications, (accepted Aug. 8, 2002).

8. D. Rappaport Computing simple circuits from a set of line segments is NP-complete, SIAM J. Comput., 18, 6, (1989) 1128-1139. 